\title{
Validation of flow impact to detect the energy loss in ball valve
}

\begin{abstract}
Researchers especially from engineering field are using Computer Fluid Dynamic (CFD) software to simulate the three dimension (3D) model before they perform any experiments to prove their theories. The aim of this study is to develop a valve model in 3D using Solidworks software and to analyze internal fluid flow using Solidworks Flow Simulation (SWF) software to prove that this software has the capability in performing fluid simulations for the ball valve. The SWF successfully calculates the value of outlet velocity for the ball valve at different closing angles and different pressures value. The results shown are significantly acceptable by referring to the outputs of practical experiment and simulation incidence's error of Ozdamar model 2007. The average error in calculation for SWF is about $7.42 \%$, unlike FLUENT which is around $11.09 \%$. Therefore; the engineers can simulate their models with higher accuracy using SWF software.
\end{abstract}

Keyword: Ball valve; Computational fluid dynamics; Solidworks flow simulations; Energy losses 